\title{
Feasibility of diffusion-weighted imaging with DWIBS in staging Hodgkin lymphoma in pediatric patients: comparison with PET/CT
}

\author{
Dobromila Baranska $^{1} \cdot$ Katarzyna Matera ${ }^{1}$ (D) Michal Podgorski ${ }^{1} \cdot$ Magdalena Gorska-Chrzastek $^{2}$. \\ Karolina Krajewska ${ }^{3}$ · Joanna Trelinska ${ }^{3}$. Piotr Grzelak ${ }^{1}$
}

Received: 11 June 2018 / Revised: 20 November 2018 / Accepted: 22 November 2018 / Published online: 29 November 2018

(c) The Author(s) 2018

\begin{abstract}
Objective The aim of the study was to evaluate feasibility of diffusion-weighted whole-body imaging with background body signal suppression (DWIBS) method in diagnosing Hodgkin lymphoma in pediatric patients and to compare it with 18F-FDG PET/CT as a gold standard.

Materials and methods Eleven patients (median age 14) with newly diagnosed Hodgkin lymphoma were examined with 18F-FDG PET/CT and MRI including whole-body DWIBS sequence $\left(b=0,800 \mathrm{~s} / \mathrm{mm}^{2}\right)$, before the oncologic treatment. About 26 locations of lymphatic tissues were evaluated visually and quantitatively using $\mathrm{ADC}_{\text {mean }}$ (DWIBS) and $\mathrm{SUV}_{\max }$ (18F-FDG PET/CT), respectively.

Results All affected lymph node regions $(n=134)$ diagnosed in 18F-FDG PET/CT were found with DWIBS, presenting decreased diffusion. Significant correlation was found between ADC and SUV values $\left(R^{2}=-0.37 ; p=0.0001\right)$. Nevertheless, additional 33 regions were recognized only by DWIBS. They were significantly smaller than regions diagnosed by both methods.
\end{abstract}

Discussion Agreement between DWIBS and 18F-FDG PET/CT for detection and staging of malignant lymphoma is high. DWIBS can be used for the evaluation of pediatric Hodgkin lymphoma.

Keywords Apparent diffusion coefficient · Diffusion-weighted magnetic resonance imaging · Hodgkin lymphoma · Positron emission tomography/computed tomography $\cdot$ Standard uptake value

\section{Introduction}

Hodgkin lymphoma (HL) incidence for children aged 15-19 had been reported as 15.9 per million. It constitutes the third most common malignancies in this age group [1]. There are several diagnostic options for the initial staging and restaging of the Hodgkin lymphoma. According to latest European recommendations (EuroNet-Pediatric

Katarzyna Matera

katarzyna.mater@gmail.com

1 Department of Diagnostic Imaging, Polish Mother's Memorial Hospital-Research Institute in Lodz, Rzgowska 281/289, 93-338 Lodz, Poland

2 PET/CT Laboratory, NZOZ MCD Voxel, Polnocna 42, 91-425 Lodz, Poland

3 Department of Pediatrics, Oncology, Hematology and Diabetology Medical, University of Lodz, Pankiewicza 16, 91-738 Lodz, Poland
Hodgkin's Lymphoma Group-C2, EuroNet-PHL-C2), the whole-body PET/MR (Positron Emission Tomography-Magnetic Resonance), both method combined with chest CT (Computed Tomography) and abdominal ultrasound are recommended. However, due to low availability, more commonly, whole-body 18F-FDG PET/CT (2-[Fluorine-18]-fluoro-2-deoxy-D-glucose positron emission tomography/computed tomography) is used [2-4]. This examination was a gold standard method in former recommendations (EuroNet-PHL-C1/Interimphase/LP1) and characterized by sensitivity and specificity for recognition of nodal disease reaching $87.5 \%$ and $85.6 \%$, respectively [5-7].

As far as the safety is concerned, in PET, the amount of radio-labeled pharmaceutical is extremely small (0.01-10 $\mu \mathrm{g})$, having essentially no pharmacological effect. However, during single 18F-FDG PET/CT examination, patient receives about $8.8 \pm 1.8 \mathrm{mSv}$. Moreover, according to actual guidelines, this examination is 
repeated in most cases 2 or 3 times a year (in the first year of disease), and then performed in all cases of suspected recurrence of the disease $[8,9]$. Even though, the CT study correlated with 18F-FDG PET has been highly optimized and is considered as a low-dose technique $[10,11]$. Contrary to this procedure, an addition of the CT examination as a separated study (e.g., chest scan) considerably increases the dose of radiation [12]. Thus, high cumulative effective dose (reaching $60-113 \mathrm{mSv}$ ) carries a risk of long-term radiation complications, especially for pediatric patients $[13,14]$.

The limitation of the $18 \mathrm{~F}-\mathrm{FDG}$ PET/CT technique is its moderate specificity, with many false-positive foci in the intestinal region associated with benign conditions such as reactive lymphatic hyperplasia, inflammation or red cell senescence [15]. With more children surviving lymphomas, finding safer and less toxic diagnostic options is a must to reduce the risk of late radiation complications such as secondary cancer and inheritable DNA mutations $[16,17]$.

Diffusion-weighted whole-body imaging with background body signal suppression (DWIBS) may be a good, radiation-free alternative $[18,19]$. This method is based on the measurements of Brownian motion of water molecules in biological tissue. In many pathological conditions, water diffusivity is impeded (low) due to, e.g., increased neoplastic cellularity or swelling in inflammatory or infectious lesions. It also concerns lymphomas, where cells are densely packed and randomly organized, inhibiting an effective motion of extracellular water [20-23]. DWIBS provides cross-sectional imaging of the entire body, with a high soft-tissue contrast, and functional information [24]. It is already employed in the follow-ups of patients with lymphoma [25-27]. It is included in EuroNet-PHL-Interimphase trial, which is still being continued by some European countries. Due to high spatial resolution, this technique allows for evaluation of the nodal space even in children, and thus accurately determining lymphoma involvement [28, 29].

There are still little data on the accuracy of DWIBS technique in initial staging of Hodgkin lymphoma in pediatric patients $[19,20,28,30,31]$. Thus, this study aims to compare the accuracy of lymphatic regions recognition between DWIBS technique and the 18F-FDG PET/CT as a gold standard in a group of pediatric patients newly diagnosed with Hodgkin lymphoma.

\section{Materials and methods}

\section{Patients}

In 11 patients newly diagnosed with Hodgkin lymphoma ( 8 girls and 3 boys, median of age 14, range 8-16 years), who followed a standard diagnostic EuroNET protocol with 18F-FDG PET/CT examination, we performed DWIBS study in short time intervals (3 days). Newly diagnosed, histologically proven lymphoma and age below 18 years were inclusion criteria. Besides, each patient with a stage $>$ IIA underwent bone marrow biopsy to determine the bone marrow involvement. All visible groups of lymphatic nodes were included in the analysis (with the exclusion of extra-nodal lesions). The study protocol was approved by a local bioethical committee (decision number 65/2017) and was in accordance with the Declaration from Helsinki. Informed consent was obtained from all the individual participants included in the study.

\section{F-FDG PET/CT procedure}

18F-FDG PET/CT procedure was performed using a PET/ CT (Discovery iQ 4-Ring, General Electric Healthcare Milwaukee, WI, USA) scanner. Images were acquired in caudal-cranial direction from the proximal one-third of the thigh to the skull base.

After at least $6 \mathrm{~h}$ of fasting (the serum glucose level was $<130 \mathrm{mg} / \mathrm{dl}$ in all patients [32]) and 60-65 min before PET imaging, each patient was injected with $3 \mathrm{~mL}$ of the 18F-FDG solution (2.5 MBq/kg of body weight). CT was performed immediately before PET with the patient in the same position. PET was 3 min per bed position (about $20 \mathrm{~cm}$ in length). The total examination time was about 10 min. The FDG uptake can be evaluated semiquantitatively using the standardized uptake value (SUV). The SUV is the activity in the lesion in $\mathrm{MBq} / \mathrm{mL}$ corrected for the weight of the patient and the dose of administered FDG.

PET, CT, and fused 18F-FDG PET/CT images were analyzed on a workstation (Intellispace Portal Workstation v7.03, Philips Healthcare Nederland) to evaluate the maximum SUV ( $\left.\mathrm{SUV}_{\max }\right)$ [33]. 3D ROI was placed over each lesion. Malignancy staging and delineation were performed by visual assessment of MIP images, multiplanar views (axial, coronal and sagittal) of PET, and fused 18F-FDG PET/CT images [34]. The lesion's SUV $_{\max }$ with each visible 18F-FDG uptake, among all foci was identified [35]. This can be achieved by satisfied spatial resolution, which in IQ-type scanners is approached by a specific detectors structure. In this apparatus model, the spatial resolution could range from $4.2 \mathrm{~mm}$ at $1 \mathrm{~cm}$ to $8.5 \mathrm{~mm}$ at $20 \mathrm{~cm}$ [36]. Analysis process was determined by reconstruction algorithms (View Point HD with point spread function modeling and Q.Clear). This Q.Clear reconstruction improves the PET image quality, with higher recovery coefficients and lower background variability [37, 38]. 
For the purpose of correlation between the Whole-Body (WB) MRI and 18F-FDG PET/CT, a lesion-by-lesion analysis was performed. $\mathrm{SUV}_{\max }$ was recorded from the largest uptake area visible in PET. Contouring was performed by radiologist with 10 years of experience in reporting PET/CT. The lowest mean apparent diffusion coefficient $\left(\mathrm{ADC}_{\text {mean }}\right)$ of ROI was generated from the ADC map.

\section{Diffusion-type WB MRI technique for staging Hodgkin lymphoma}

All examinations were performed using a 1.5-T MRI system (Ingenia Omega HP, Philips Healthcare Nederland) equipped with a torso coil (dStream Torso, 32 channels, Philips Healthcare Nederland), head and neck coil (dStream HeadNeck, 16 channels, Philips Healthcare Nederland) to cover head, neck and trunk [38]. Two age-dependent protocols were prepared for patients younger than 10 years and older than 11 years. The protocol was (or The protocols were) split over (a/the) field of view covering patient's body from skull base to mid thighs (as designed in EuroNet-PHL protocols). WB MRI exam consisted of coronal T1-weighted turbo spin-echo (T1W_TSE) sequence with breath-holding in chest and abdomen, coronal fat-suppressed T2-weighted (T2W_STIR) short tau inversion recovery (STIR) with respiratory triggering in chest and abdomen. Diffusion-type WB MRI was performed with DWIBS using echo planar imaging (EPI) during free breathing [4]. Each listed sequence was equipped with parallel acquisition technique (sensitivity encoding, SENSE), which is responsible for reaching an increased spatial resolution and decrease acquisition time. Thus, application of SENSE makes WB MRI examination an adjusted tool for reaching an excellent softtissue contrast $[5,39,40]$. Double $b$ values were selected for ADC calculation, 0 and 800 [41]. Coronal maximum intensity projection (MIP) images were reconstructed from STIR and DWIBS separately. The MIP thickness was displayed and calculated automatically from the patient's axial or coronal field of view. In addition, an axial turbo spin-echo (T1W_TSE) scan was also included for further characterization of suspected lesions. The examinations were performed without contrast agent administration. Total examination time was estimated for about $35 \mathrm{~min}$. Table 1 shows the sequences used in protocol.

\section{Evaluation of data correlation}

It should be initially noted that there is no generally valid WB MRI protocol; thus, for the study, the original total body protocol was created. However, until now, different combinations have been used to formulate the most matched protocol for lymphomas [28, 42]. In our study, WB MRI evaluation began by reviewing the MobiView of the T2W_STIR, followed by the correlation with the axial DWIBS and axial morphologic images.

In lymph nodes evaluation, we assimilated EuroNet-PHL recommendations for CT examination. Thus, lymph nodes were recognized in the T2W_STIR sequence as positive

Table 1 WB MRI sequence protocol parameters

\begin{tabular}{|c|c|c|c|c|}
\hline Parameter & T2W_TSE & DWIBS & T1W_TSE & T2W_STIR \\
\hline Echo & SE fast imaging mode TSE Multishot & $\begin{array}{l}\text { IR fast imaging mode EPI } \\
\text { Single-shot double b-factors }\end{array}$ & $\begin{array}{l}\text { IR fast imaging } \\
\text { mode EPI Mul- } \\
\text { tishot }\end{array}$ & $\begin{array}{l}\text { IR fast } \\
\text { imaging } \\
\text { mode TSE } \\
\text { Single-shot }\end{array}$ \\
\hline $\mathrm{TR}(\mathrm{ms})$ & $12021 / 13023^{*}$ & $6260 / 6335^{*}$ & $11281 / 11986^{*}$ & 5.4 \\
\hline $\mathrm{TE}(\mathrm{ms})$ & 100 & $65 / 67 *$ & 70 & $1.72 / 3.6$ \\
\hline Flip angle $\left(^{\circ}\right)$ & 90 & No & No & 15 \\
\hline NSA & 2 & 4 & 1 & 1 \\
\hline Slice thickness $(\mathrm{mm})$ & 3 & 5 & 5 & 2.2 \\
\hline Slice gap (mm) & 0.8 & 1 & 0.7 & 0 \\
\hline WFS & Maximum & Minimum & Maximum & Default \\
\hline SENSE & Yes & Yes & Yes & Yes \\
\hline Max $b$ factor & No & 800 & No & No \\
\hline Breath hold & No & No & Expiration & Expiration \\
\hline Phase encoding & RL & $\mathrm{AP}$ & RL & RL \\
\hline Estimated time (mm:ss) & $04: 13 / 05: 00^{*}$ & 03:02/03:04* & $05: 24$ & $00: 34 / 00: 36^{*}$ \\
\hline Number of sequence used & $2 \times($ chest, head and neck $)$ & $4 \times($ head, neck, chest, abdomen) & $1 \times$ chest & $\begin{array}{l}4 \times(\text { head, } \\
\text { neck, chest, } \\
\text { abdomen })\end{array}$ \\
\hline
\end{tabular}

Patient age: up to 10

* Above 11 years old 
(lymphomatous) when any diameter was $>2 \mathrm{~cm}$, suspicious when it ranged between 1 and $2 \mathrm{~cm}$, and negative if it was $<1 \mathrm{~cm}$. Additionally, in this study, a lymph node was suspected of malignancy if it showed a focal signal intensity equal to or greater than the organ with usually highest diffusion signal intensity in the same region (brain, salivary glands, tonsils, spleen, gallbladder, adrenal glands, prostate, spinal cord, peripheral nerves, bone marrow and reproductive organs) despite the size [18, 42]. Lymph nodes were not measured on diffusion-weighted images because the measurements are highly dependent on the applied window level and window width. Instead, diffusion-weighted images were used only to detect potential nodal abnormalities and to estimate ADC values. Also, any pathological signals from DWIBS had to be confirmed by morphological scans.

After confirming the node size in the STIR image, a freehand region of interest (ROI) measurement with ellipsoidal contour was then drawn on DWIBS (b800) image to encompass the entire cross section of the lesion. ADC of the lymph node was performed on the ADC map, automatically. Each visible lymph node was measured on one slice, on which it appeared to be the largest. Mean ADC and ellipse surface area of each lesion were assessed. The high coverage achieved by DWIBS allows to classify disease to one of the IV spreading stages [30]. We used Ann Arbor scale to classify the lymphoma and refer it to the staging performed based on 18F-FDG PET/CT.

\section{Statistical analysis}

In statistical analysis, we had two goals. Firstly, to assess whether the DWIBS examination allowed to recognize affected regions with the same accuracy as the 18F-FDG PET/CT. For analysis, we used the Chi square test. The Mann-Whitney test was used to compare size of lesions between two groups. Secondly, to evaluate the association between the $\mathrm{SUV}_{\max }$ the $\mathrm{ADC}_{\text {mean }}$, particularly/mainly, to check whether the $\mathrm{ADC}$ can be used as a quantitative surrogate of neoplastic process aggravation. We evaluated normality of data distribution with the Shapiro-Wilk test and the due to not-normally distributed variables, we applied Spearman's rank correlation test. In addition, this test enabled to evaluate a correlation between an area of the lymph node and $\mathrm{ADC}_{\text {mean }}$. The statistical analysis was performed using Statistica 12 software (StatSoft Polska, Cracow, Poland). A $p$ value below 0.05 was considered significant. The results are presented as a mean and standard deviation.

\section{Results}

All patients were diagnosed with multiple locations of neoplastic process (median number of 16 locations, range 7-26). Lesions with pathologic features in both imaging methods were termed as recognized regions. The lesion's $\mathrm{SUV}_{\max }$ with each visible 18F-FDG uptake, among all foci was identified. For correlation between apparent diffusion coefficient (ADC) and 18F-FDG quantification, a lesionby-lesion analysis was performed. In this analysis, $\mathrm{SUV}_{\max }$ was recorded for the lesion with the largest diameter and the lowest mean apparent diffusion coefficient $\left(\mathrm{ADC}_{\text {mean }}\right)$ value on the WB MRI (Fig. 1). Due to a better spatial resolution of MRI examination comparing to the 18F-FDG PET/CT, it was possible to delineate nodal groups better and qualify into anatomical regions properly, for instance, not to mediastinal but to axillar or subclavicular region. All regions of increased metabolism visible in 18F-FDG PET/CT presented decreased diffusion in DWIBS. Moreover, values of $\mathrm{ADC}_{\text {mean }}$ and $\mathrm{SUV}_{\text {max }}$ correlated significantly with each other $\left(R^{2}=-0.36 ; p=0.0002\right)$ (Fig. 2).

Nevertheless, 33 regions with restricted diffusion were not recognized in $18 \mathrm{~F}-\mathrm{FDG} \mathrm{PET} / \mathrm{CT}$. These regions were significantly smaller $\left(35.2, \mathrm{SD}=30.9 \mathrm{~mm}^{2}\right)$ than localizations visible in both techniques $\left(86.2 \mathrm{SD}=70.3 \mathrm{~mm}^{2}\right)$ $(p=0.0001)$. There was a significant negative relationship between the lymph node area and the ADC value, which is confirmed by the Spearman's rank correlation coefficient $\left(R^{2}=-0.321, p<0.001\right)$ (Fig. 3). Detailed information concerning occupied regions together with their SUV and ADC values are presented in Table 2 .

Conventional staging procedures were performed as clinically indicated and as recommended by current oncologic protocols. According to the Ann Arbor staging system using 18F-FDG PET/CT and bone marrow biopsy, the disease was stage II (type A, E) in eight patients, in remaining: three patients, stages IIIB, IA and IVA were classified. Classification performed based on WB MRI confirmed results of $18 \mathrm{~F}-\mathrm{FDG}$ PET/CT in nine patients; while in remaining two, it resulted in overstaging.

\section{Discussion}

We showed that the WB MRI and DWIBS techniques allow to recognize FDG-avid lymph nodes and have excellent agreement with the 18F-FDG PET/CT. So far, only two studies (Punwani et al. [41] and Kwee et al. [43]) showed similar results for children. However, what is unique about our study is the narrow group of patients: children with the initial diagnosis of particular type of lymphoma (Hodgkin's). We assumed that the different types of lymphoma might vary in types of DWIBS findings. Thus, to make the comparison with 18F-FDG PET/CT more reliable, we focused on a homorganic group of great importance. This importance is due to the young age, resulting in an increased risk of future complications due to diagnostics (e.g., radiation) and treatment. 


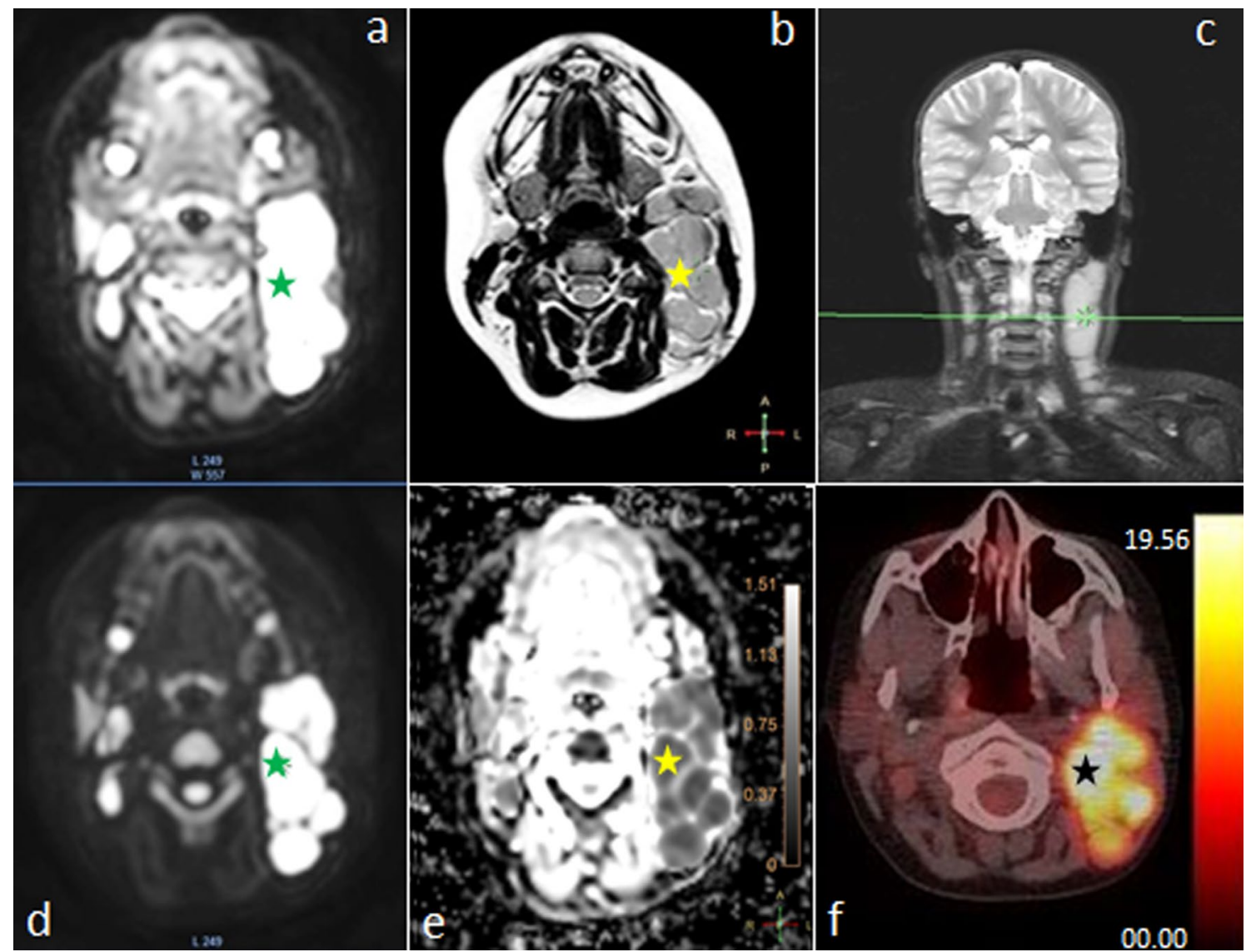

Fig. 1 Images of a 14-year-old girl with Hodgkin lymphoma, showing the same enlarged cervical group of lymph nodes: a WB MRI, axial of b0 DWIBS image hyperintensity restricted diffusion (green star), b WB MRI, axial of T2-weighted image with isointensity region (yellow star); $\mathbf{c}$ WB MRI, coronal MIP reconstruction of head and neck STIR image with high signal intensity region (little green

Presented research assessed the WB MRI capabilities to recognize and to perform grading in patients with Hodgkin's disease. We showed that the WB MR and DWIBS are able not only to confirm the 18F-FDG PET/CT findings but also to inspect more of the lesions than hybrid PET/CT. Based on our research, it can be hypothesized that significant correlation between $\mathrm{ADC}_{\text {mean }}$ and $\mathrm{SUV}_{\text {max }}$ indicates that DWIBS may allow to quantitatively assess the disease aggravation. However, due to only fair level of correlation between SUV and ADC, further studies will need to confirm this assumption. At the same time, $\mathrm{ADC}_{\text {mean }}$ and $\mathrm{SUV}_{\text {max }}$ were considered as the most reliable, due to the relationship between these metrics, that has been clarified and positively evaluated by some researchers in other lymphoma subtypes $[29,41$, $43,44]$.

A comparison of our results with the other researches is difficult because there is no other study on comparable Hodgkin's lymphoma cohort as other studies differ in the applied $\mathrm{b}$ parameters, the age of included individuals and cross on green, horizontal line); d WB MRI, axial of b800 DWIBS image subtraction with restricted diffusion region (green star); $\mathrm{e}$ WB MRI, axial of ADC map from DWIBS images subtraction with hypointensity restricted diffusion region (yellow star) and ADC scale; f $18 \mathrm{~F}-\mathrm{FDG}$ PET/CT axial image showing $18 \mathrm{~F}-\mathrm{FDG}$ uptake (black star) of cervical region and SUV scale

the types of examined neoplasms [26, 45]. The meta-analysis by Regacini et al. analyzing cases reports between 2010 and 2013 demonstrated the high specificity of both methods in the primary staging of lymphomas (59-100\% of lymph nodes diagnosed with 18F-FDG PET/CT were confirmed with the WB MRI). In addition, it showed the compatibility of these techniques in over $90 \%$ [46]. Furthermore, in region-specific analysis, our research confirms the high compliance of coverage of areas in both methods, which is not as good as shown by Albano et al. and Ferrari et al. but occurs greater than in van Ufford et al. paper [47-49]. One of the causes of discrepancies might be a heterogeneous composition of patients groups included in cited studies.

On the other hand, Mosavi et al. [29] reported a lack of compliance between 18F-FDG PET/CT and the WB MRI in the Hodgkin's lymphoma patients. This lack of compliance may be due to the selection of another age group (17-78 years). There is also no systematic information on whether patients were newly diagnosed. This fact is 
Fig. 2 Correlations between $\mathrm{ADC}_{\text {mean }}$ and $\mathrm{SUV}_{\text {max }}$ variables

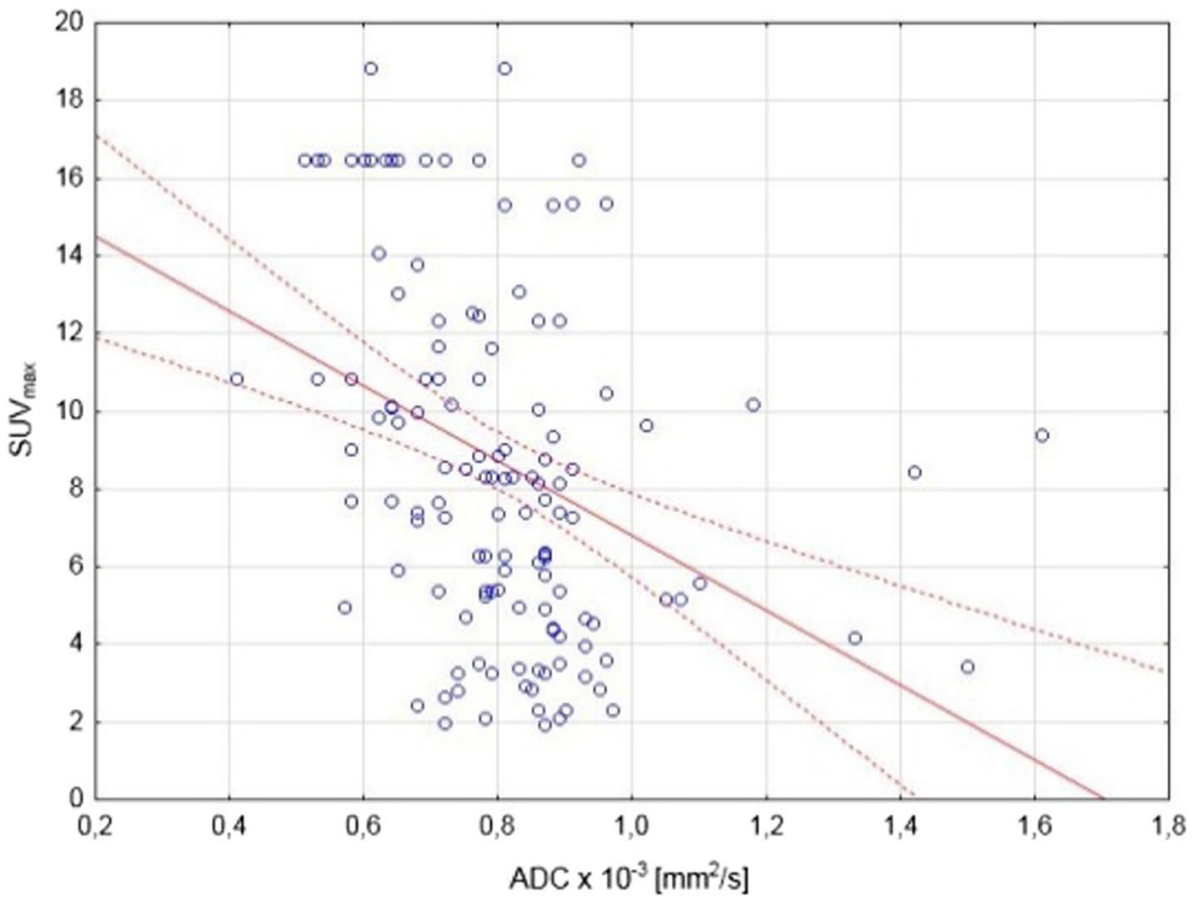

Fig. 3 Scatter diagram with regression line occurs lymph node area $(S)$ and $\mathrm{ADC}_{\text {mean }}$ value dependence

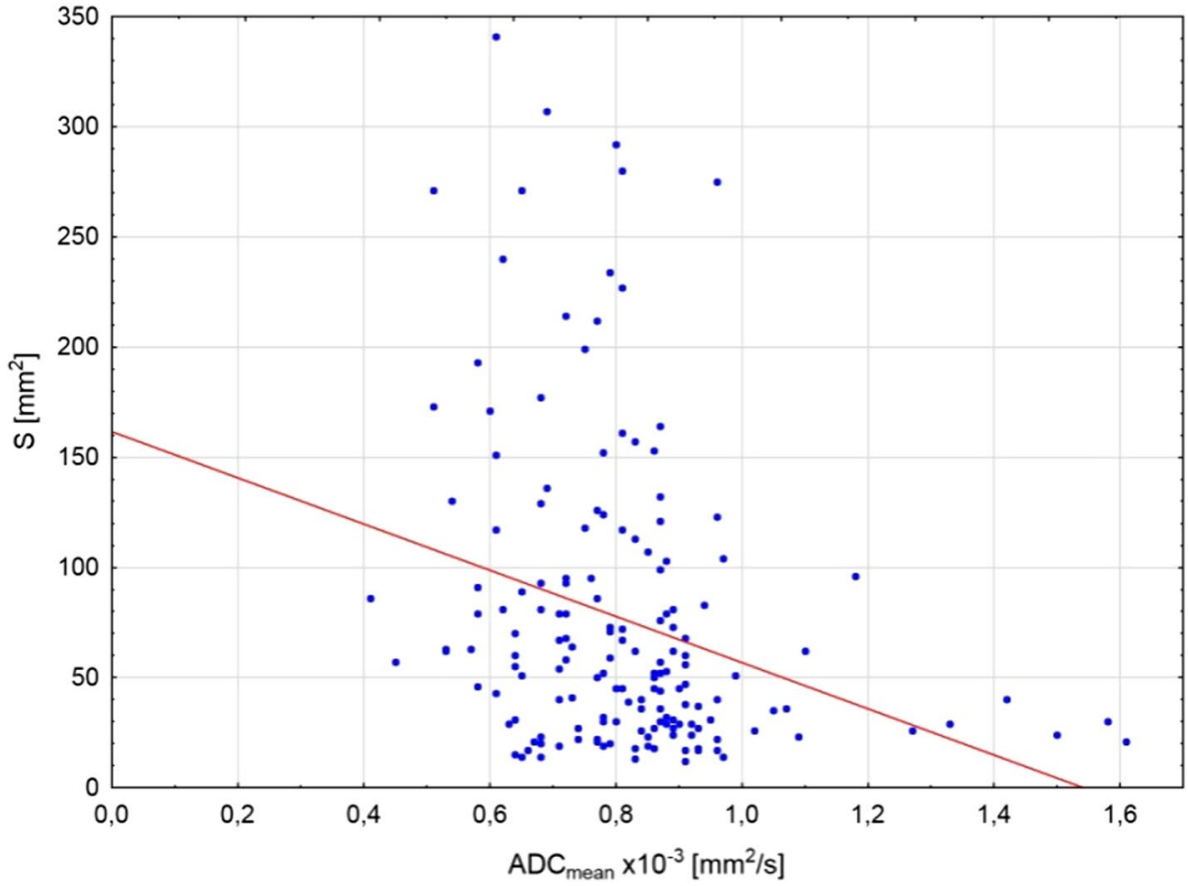

important because children's lymphomas are characterized by a specific course and symptoms, unlike adults ones [4]. The last meta-analysis [50], which reported lack of significant relationship between ADC and SUV parameter, explains the variances throughout the biology of different neoplasms [50].

Disparities between DWIBS and 18F-FDG PET/CT are clinically important because they may affect staging of the disease. In our research, $19.76 \%$ of areas were diagnosed only by DWIBS which resulted in overstaging in 2 out of 11 patients. In contrast, on $\mathrm{Gu}$ et al. [51], overstaging concerns $11.8 \%$ of patients; whereas in the research of Abdulqadhr et al. [52] it is only $9.7 \%$.

Furthermore, Stéphane et al. [25] showed that there might be no over-interpretation of DWIBS and that $100 \%$ classification coverage in both methods is possible. It should be 
Table 2 The counts of the identified areas in the two methods of PET/CT and DWIBS, including measured coefficients of SUV and ADC, respectively

\begin{tabular}{lclll}
\hline Localization/region & DWIBS & $\begin{array}{l}\text { ADC } \times 10^{-3}\left(\mathrm{~mm}^{2} / \mathrm{s}\right) \\
(\mathrm{SD})\end{array}$ & PET/CT & SUV (unitless) (SD) \\
\hline Cervical & 37 & $0.74(0.14)$ & 30 & $10.01(5.49)$ \\
Palatine tonsil & 22 & $0.78(0.14)$ & 22 & $7.21(2.56)$ \\
Axilla & 20 & $0.82(0.09)$ & 12 & $6.62(2.64)$ \\
Supraclavicular & 18 & $0.81(0.21)$ & 15 & $9.64(4.39)$ \\
Subclavicular & 18 & $0.83(0.26)$ & 15 & $8.27(4.15)$ \\
Mediastinum & 16 & $1.00(0.27)$ & 14 & $10.14(4.00)$ \\
Submandibular & 13 & $0.84(0.16)$ & 7 & $6.12(6.23)$ \\
Paratracheal & 7 & $0.86(0.04)$ & 7 & $11.54(5.20)$ \\
Paraaortic & 6 & $0.74(0.17)$ & 6 & $10.65(4.14)$ \\
Maxillary & 4 & $0.79(0.04)$ & 2 & $3.18(0.47)$ \\
Parotid & 2 & $0.86(0.04)$ & 2 & $3.46(0.07)$ \\
Visceral & 2 & $0.90(0.09)$ & 1 & 13.07 \\
Inguinal & 2 & $0.80(0.11)$ & 1 & 1.95 \\
Total & 167 & & 134 & \\
\hline
\end{tabular}

noticed that in both of these two overstaged cases, additional locations of affected lymph nodes were recognized below the level of the diaphragm. Thus, observed discrepancies were not due to the differences in patient positioning (in the MRI study, patients were placed with hands along the body, while in the 18F-FDG PET/CT, hands were placed behind). Interestingly, pathomorphological evaluation confirmed the nodular lymphocyte-predominant Hodgkin's lymphoma type in both patients. In this subtype, lymph nodes are densely packed with lymphocytes; thus, diffusion might be profoundly imparted [53]. It may indicate that DWIBS might be a better method for diagnosing patients with this subtype of lymphoma. Nevertheless, further studies including a larger group of patients are required.

Another reason for overstaging may consider the size of analyzed nodules. Overstaging is a consequence of additional lymph nodules recognized in MRI. Additional lymph nodules were significantly smaller than those recognized in both techniques. From the analytic point of view, these additional findings might be treated as the false-positive ones. However, morphological scans and initial follow-up (data not presented) indicate that they were affected. Thus, it rather supports the theory that WB MRI has higher accuracy than 18F-FDG PET/CT.

Finally, discrepancies between DWIBS and 18F-FDG PET/CT might be caused by a lack of ADC diagnostic standards. There are no ADC criteria for differentiating small lymph nodes with low ADC from the same sized, healthy nodes (with no significant diffusion restriction) [19]. On the other hand, the ADC is the only technique that allows for evaluating signal intensity criteria for discriminating between lymphomatous and normal lymph nodes. Nevertheless, it is only reported that ADC is lowest in lymphomatous nodes and highest in healthy ones [31].
Taking into account all the above, the critical task for making WB MRI with DWIBS technique a reliable diagnostic method is to determine diagnostic references of ADC [25, 54]. Our analysis showed that in all affected lymph nodes, the apparent diffusion coefficient was lower than $1 \times 10^{-3} \mathrm{~mm}^{2} / \mathrm{s}$. It is only the simple fact, without further conclusions considering this value as a cut point on the ADC scale. Additional researches on a large cohort are required to determine referential values. Moreover, the technique of ADC measurement should be unified. Measurement of an ADC value is somewhat challenging and may vary between different centers because of the different ADC measurement parameters. The ADC measurement can also be negatively affected by tissue heterogeneity and respiratory artifacts [55]. According to Kwee and Takahara, free breathing is referred to as the "driving force" in the DWIBS sequence [56]. Nevertheless, they pointed out that diaphragm movement can disturb the visualization of small-sized lymph nodes placed around the spleen or liver [44]. In a more recent study, Stone et al. [57] designated that mean ADC values are not much affected by breathing, but there is a significant increase in the spread of standard deviation of ADC values, what may be attributed to blurring effects. The solution may be to use Navigator-based triggering or other tracking systems [58].

In summary, presented results indicated that the mean ADC can potentially discriminate between healthy and lymphomatous lymph nodes. Also, DWIBS could be a useful, non-invasive and radiation-free functional imaging method for visualizing lymphomatous lesions in children. Furthermore, ADC can be used for a quantitative evaluation of process aggravation [59]. Thus, ADC might become a biomarker of the Hodgkin's lymphoma course and a diagnostic tool of choice instead of single CT examination [20, 30]. 
Thus, the use of DWIBS instead of single CT may initiate changes in oncologic trials in the future, especially in pediatric patients.

\section{Limitations}

The first limitation of present study is lack of comparison between ADC and SUV in extranodal regions. Organs which can be affected by lymphoma are liver, uterus, skeletal system and bone marrow. Analysis of extranodal regions is mandatory to properly classify lymphoma according to Ann Arbor system. Despite the fact that our group of patients consisted of those who were mainly classified in the second stage of disease (no extranodal regions affected) and that the bone marrow involvement does not frequently appear among Hodgkin lymphoma patients, measuring the ADC parameter in all extranodal structures has a diagnostic potential [60] and would be performed in our future study. So far, PET examination was the one which affirmed bone marrow involvement with high accuracy; however, this standard could be changed in the future in favor of diffusion-weighted imaging precision [61-64].

Secondly, despite the spatial resolution difference between WB MRI and 18F-FDG PET/CT, no direct calculation to assess these differences was performed. The role of the PET/CT examination in oncological trials is widely accepted; however, there were first reports which verified that MRI has a better ability to differentiate smaller areas than 18F-FDG PET/CT [65, 66]. We demonstrated two parameters: SUV and ADC, which correlate with spatial resolution in 18F-FDG PET/CT and MRI studies, respectively. However, quantitative assessment of the resolution would be our goal in the follow-up.

The next potential limitation comes from the presumption that the selected $b$ values of 0 and 800 are optimal for DWIBS examination in children. The ADC decrease is inversely proportional to the $b$ value [67]. Thus, there is no optimal choice of $b$ values in WB DWIBS. According to our research, the choice was made by an experienced radiologist based on the observations and general knowledge [39, 41]. We are also familiar with the paper by Koc and Erbay, where they listed the most valuable $b$ values [68]. Thus, there is a papers where b0 and b1000 or combination of three $b$ values are used. However, we decided to use b800 and b0 pair to reach optimal SNR and to gain a study time [69]. Other researches confirmed efficacy of b800 to recognize all lymph nodes with restricted diffusion $[62,66,69]$.

Another limitation concerns lack of 18F-FDG PET/CT scanner EARL-accreditation. It enables a comparison of quantitative results with other centers. Nevertheless, it does not bias our findings of the correlation between PET/CT and DWIBS.

Finally, there is a potential bias in our analysis, as we evaluated correlation between $\mathrm{ADC}_{\text {mean }} \mathrm{vs} \mathrm{SUV}_{\text {max }}$ in large number of lymph nodes, but derived only from 11 patients. Thus, outlining results in a single patent might be multiplied when evaluating all lymph nodes together. To reduce this risk, we controlled for outliers and observed homogenous trends in all patients.

\section{Conclusion}

Our study showed that the WB MRI with DWIBS technique is useful in identifying and staging affected lymph nodes in the pretreatment group of newly diagnosed children with Hodgkin's lymphoma. This method is also superior to 18F-FDG $\mathrm{PET} / \mathrm{CT}$ in recognizing small lesions. WB MRI examination, firstly, allows to obtain high-resolution diagnostic images. Secondly, it allows to perform evaluation without contrast and radiation in a relatively short time. It may shed light on future perspective to avoid additional CT scan which is now attributed to standard oncologic protocols. Thus, our initial research may give a foundation to create a universal diagnostic model dedicated to diagnosing Hodgkin's lymphoma in peadiatric patients.

Acknowledgements We thank Abhinav B. Shukla as a writing assistant for language editing.

Author contributions Study conception and design: DB, MG-C, KK, JT, PG. Acquisition of data: DB, KM, MG-C, KK, JT. Analysis and interpretation of data: DB, MP, KM. Drafting of manuscript: DB, MP, KM. Critical revision: DB, MP, PG, JT.

\section{Compliance with ethical standards}

Conflict of interest All authors declare that they have no conflicts of interest.

Ethical approval The study was performed in compliance with ethical standards.

Open Access This article is distributed under the terms of the Creative Commons Attribution 4.0 International License (http://creativeco mmons.org/licenses/by/4.0/), which permits unrestricted use, distribution, and reproduction in any medium, provided you give appropriate credit to the original author(s) and the source, provide a link to the Creative Commons license, and indicate if changes were made.

\section{References}

1. Steliarova-Foucher E, Stiller C, Kaatsch P et al (2004) Geographical patterns and time trends of cancer incidence and survival among children and adolescents in Europe since the 1970s (the ACCIS project): an epidemiological study. Lancet 364:2097-2105

2. Kluge R, Kurch L, Georgi T, Metzger M (2017) Current role of FDG-PET in pediatric Hodgkin's lymphoma. Semin Nucl Med 47(3):242-257

3. Körholz D et al (2017) Second international Inter-Group Study for classical Hodgkin's lymphoma in children and adolescents 
4. Mauz-Körholz C, Metzger ML, Kelly KM, Schwartz CL, Castellanos ME, Dieckmann K, Kluge R, Körholz D (2015) Pediatric Hodgkin lymphoma. J Clin Oncol. 33(27):2975-2985

5. Kwee TC, Kwee RM, Nievelstein RA (2008) Imaging in staging of malignant lymphoma: a systematic review. Blood 111:504-516

6. Juweid ME, Stroobants S, Hoekstra OS, Imaging Subcommittee of International Harmonization Project in Lymphoma et al (2007) Use of positron emission tomography for response assessment of lymphoma: consensus of the Imaging Subcommittee of International Harmonization Project in Lymphoma. J Clin Oncol 25:571-578

7. la Fougère $\mathrm{C}$, Hundt $\mathrm{W}$, Bröckel $\mathrm{N}$ et al (2006) Value of PET/ $\mathrm{CT}$ versus $\mathrm{PET}$ and $\mathrm{CT}$ performed as separate investigations in patients with Hodgkin's disease and non-Hodgkin's lymphoma. Eur J Nucl Med Mol Imaging 33(12):1417-1425

8. Fletcher JW, Djulbegovic B, Soares HP (2008) Recommendations on the use of 18F-FDG PET in oncology. J Nucl Med 49:480-508

9. Fahey FH, Goodkind A, MacDougall RD, Oberg L, Ziniel SI, Cappock R, Callahan MJ, Kwatra N, Treves ST, Voss SD (2017) Operational and dosimetric aspects of pediatric PET/CT. J Nucl Med 58(9):1360-1366

10. Parisi MT, Bermo MS, Alessio AM, Sharp SE, Gelfand MJ, Shulkin BL (2017) Optimization of pediatric PET/CT. Semin Nucl Med 47(3):258-274

11. Marafi F, Esmail A, Rasheed R, Alkandari F, Usmani S (2017) Novel weight-based dose threshold for $18 \mathrm{~F}-\mathrm{NaF}$ PET-CT imaging using advanced PET-CT systems: a potential tool for reducing radiation burden. Nucl Med Commun 38(9):764-770

12. Brenner DJ, Hall EJ (2007) Computed tomography—an increasing source of radiation exposure. N Engl J Med 357:2277-2284

13. Pierce DA, Preston DL (2000) Radiation-related cancer risks at low doses among atomic bomb survivors. Radiat Res 154(2):178-186

14. Nievelstein RA, Quarles van Ufford HM, Kwee TC et al (2012) Radiation exposure and mortality risk from CT and PET imaging of patients with malignant lymphoma. Eur Radiol 22(9):1946-1954

15. Kabickova E, Votrubova J, Sumerauer D, Mejstrikova E, Hrusak O, Kyncl M, Kodet R, Stary J, Belohlavek O (2009) Diagnostic value and limitations of FDG-PET for the staging of childhood lymphomas. Blood 114:3658

16. Brenner D, Elliston C, Hall E, Berdon W (2001) Estimated risks of radiation-induced fatal cancer from pediatric CT. AJR Am J Roentgenol 176:289-296

17. Kleinerman RA (2006) Cancer risks following diagnostic and therapeutic radiation exposure in children. Pediatr Radiol $36: 121-125$

18. Kwee TC, Takahara T, Ochiai R, Nievelstein RA, Luijten PR (2008) Diffusion-weighted whole-body imaging with background body signal suppression (DWIBS): features and potential applications in oncology. Eur Radiol 18(9):1937-1952

19. Nievelstein RA, Littooij AS (2016) Whole-body MRI in paediatric oncology. Radiol Med 121(5):442-453

20. Littooij AS, Kwee TC, Barber I, Granata C, Vermoolen MA, Enríquez G, Zsíros J, Soh SY, de Keizer B, Beek FJ, Hobbelink MG, Bierings MB, Stoker J, Nievelstein RA (2014) Whole-body MRI for initial staging of paediatric lymphoma: prospective comparison to an FDG-PET/CT-based reference standard. Eur Radiol 24(5):1153-1165

21. Zink D, Fischer AH, Nickerson JA (2004) Nuclear structure in cancer cells. Nat Rev Cancer 4(9):677-687

22. Zheng W, Aspelund A, Alitalo K (2014) Lymphangiogenic factors, mechanisms, and applications. J Clin Investig 124(3):878-887

23. Uhl M, Altehoefer C, Kontny U, Il'yasov K, Büchert M, Langer M (2002) MRI-diffusion imaging of neuroblastomas: first results and correlation to histology. Eur Radiol 12(9):2335-2338
24. Holzapfel K, Duetsch S, Fauser C, Eiber M, Rummeny EJ, Gaa J (2009) Value of diffusion-weighted MR imaging in the differentiation between benign and malignant cervical lymph nodes. Eur Radiol 72(3):381-387

25. Stéphane V, Samuel B, Vincent D, Joelle G, Remy P, Francois GG, Jean-Pierre T (2013) Comparison of PET-CT and magnetic resonance diffusion weighted imaging with body suppression (DWIBS) for initial staging of malignant lymphomas. Eur J Radiol 82(11):2011-2017

26. Wong CS, Gong N, Chu YC, Anthony MP, Chan Q, Lee HF, Chu KM, Khong PL (2012) Correlation of measurements from diffusion weighted MR imaging and FDG PET/CT in GIST patients: ADC versus SUV. Eur J Radiol 81(9):2122-2126

27. Punwani S, Taylor SA, Bainbridge A et al (2010) Pediatric and adolescent lymphoma: comparison of whole-body STIR halfFourier RARE MR imaging with an enhanced PET/CT reference for initial staging. Radiology 255:182-190

28. Kellenberger CJ, Miller SF, Khan M, Gilday DL, Weitzman S, Babyn PS (2004) Initial experience with FSE STIR wholebody MR imaging for staging lymphoma in children. Eur Radiol 14:1829-1841

29. Mosavi F, Wassberg C, Selling J, Molin D, Ahlström H (2015) Whole-body diffusion-weighted MRI and (18)F-FDG PET/CT can discriminate between different lymphoma subtypes. Clin Radiol 70(11):1229-1236

30. Kwee TC, Takahara T, Vermoolen MA, Bierings MB, Mali WP, Nievelstein RA (2010) Whole-body diffusion-weighted imaging for staging malignant lymphoma in children. Pediatr Radiol 40(10):1592-1602

31. Kirchner J, Deuschl C, Schweiger B, Herrmann K, Forsting M, Buchbender C, Antoch G, Umutlu L (2017) Imaging children suffering from lymphoma: an evaluation of different 18F-FDG PET/ MRI protocols compared to whole-body DW-MRI. Eur J Nucl Med Mol Imaging 44(10):1742-1750

32. Klenk C, Gawande R, Uslu L et al (2014) Ionising radiation-free whole-body MRI versus $18 \mathrm{~F}$-fluorodeoxyglucose PET/CT scans for children and young adults with cancer: a prospective, nonrandomised, single-centre study. Lancet Oncol 15(3):275-285

33. Lina C, Lucianib A, Ittic E, Haiound C, Safard V, Meignanc M, Rahmounib A (2012) Whole-body diffusion magnetic resonance imaging in the assessment of lymphoma. Cancer Imaging 12(2):403-408

34. Maccioni F, Cardinale J, Al Ansari N, Brocchieri S, Buonocore V, Catalano C (2015) Diagnostic value of MR-DWI technique in the diagnosis and staging of Hodgkin and non-Hodgkin lymphoma: comparison with PET-CT methods. In: Poster No.: C-2106, ECR 2015, scientific exhibition

35. Stecco A, Buemi F, Quagliozzi M et al (2015) Staging of primary abdominal lymphomas: comparison of whole-body MRI with diffusion-weighted imaging and ${ }^{18}$ F-FDG-PET/CT. Gastroenterol Res Pract 2015:104794

36. Kwee TC, de Klerk JMH, Nievelstein RAJ (2011) Imaging of bone marrow involvement in lymphoma: state of the art and future directions. Sci World J 11:391-402

37. Reynés-Llompart G, Gámez-Cenzano C, Romero-Zayas I, Rodríguez-Bel L, Vercher-Conejero JL (2017) Performance characteristics of the whole-body discovery IQ PET/CT system. J Nucl Med 58(7):1155-1161

38. Teoh EJ, McGowan DR, Macpherson RE, Bradley KM, Gleeson FV (2015) Phantom and clinical evaluation of the Bayesian penalized likelihood reconstruction algorithm Q.Clear on an LYSO PET/CT system. J Nucl Med 56:1447-1452

39. Lauenstein TC, Semelka RC (2006) Emerging techniques: wholebody screening and staging with MRI. J Magn Reson Imaging 24:489-498 
40. Ladd SC, Zenge M, Antoch G, Forsting M (2006) Whole-body MR diagnostic concepts. Rofo 178:763-770

41. Punwani S, Prakash V, Bainbridge A, Taylor SA, Bandula S, Olsen OE, Hain SF, Shankar A, Daw S, Humphries P (2010) Quantitative diffusion weighted MRI: a functional biomarker of nodal disease in Hodgkin lymphoma? Cancer Biomark 7(4):249-259

42. Zijlstra JM, Comans EF, van Lingen A (2007) FDG PET in lymphoma: the need for standardization of interpretation. An observer variation study. Nucl Med Commun 28(10):798-803

43. Kwee TC, Ludwig I, Uiterwaal CS et al (2011) ADC measurements in the evaluation of lymph nodes in patients with nonHodgkin lymphoma: feasibility study. Magn Reson Mater Phy 24:1

44. Takahara T, Imai Y, Yamashita T, Yasuda S, Nasu S, Van Cauteren M (2004) Diffusion weighted whole body imaging with background body signal suppression (DWIBS): technical improvement using free breathing, STIR and high resolution 3D display. Radiat Med 22:275-282

45. Mosavi F, Ullenhag G, Ahlström H (2013) Whole-body MRI including diffusion-weighted imaging compared to CT for staging of malignant melanoma. Upsala J Med Sci 118(2):91-97

46. Regacini R, Puchnick A, Shigueoka DC, Iared W, Lederman HM (2015) Whole-body diffusion-weighted magnetic resonance imaging versus FDG-PET/CT for initial lymphoma staging: systematic review on diagnostic test accuracy studies. Sao Paulo Med J 133(2):141-150

47. Albano D, Patti C, La Grutta L et al (2016) Comparison between whole-body MRI with diffusion-weighted imaging and PET/CT in staging newly diagnosed FDG-avid lymphomas. Eur J Radiol 85:313-318

48. Ferrari C, Minoia C, Asabella AN, Nicoletti A, Altini C, Antonica F, Ficco M, Guarini A, Maggialetti N, Rubini G (2014) Whole body magnetic resonance with diffusion weighted sequence with body signal suppression compared to (18)F-FDG PET/CT in newly diagnosed lymphoma. Hell J Nucl Med 17(Suppl 1):40-49

49. van Ufford HM, Kwee TC, Beek FJ et al (2011) Newly diagnosed lymphoma: initial results with whole-body T1-weighted, STIR, and diffusion-weighted MRI compared with 18F-FDG PET/CT. AJR Am J Roentgenol 196(3):662-669

50. Deng S, Wu Z, Wu Y, Zhang W, Li J, Dai N et al (2017) Metaanalysis of the correlation between apparent diffusion coefficient and standardized uptake value in malignant disease. Contrast Media Mol Imaging 2017:4729547

51. Gu J, Chan T, Zhang J et al (2011) Whole-body diffusion-weighted imaging: the added value to whole-body MRI at initial diagnosis of lymphoma. AJR Am J Roentgenol 197(3):W384-W391

52. Abdulqadhr G, Molin D, Aström G et al (2011) Whole-body diffusion-weighted imaging compared with FDG-PET/CT in staging of lymphoma patients. Acta Radiol 52(2):173-180

53. Anderson JR, Tumours I (1985) General features, types and examples. In: Anderson JR (ed) Muir's textbook of pathology, 20th edn. Edward Arnold, London, pp 121-149

54. Komori T, Narabayashi I, Matsumura K, Matsuki M, Akagi H, Ogura Y, Aga F, Adachi I (2007)
2-Fluorine-18]-fluoro-2-deoxy-D-glucose positron emission tomography/computed tomography versus whole-body diffusionweighted MRI for detection of malignant lesions: initial experience. Ann Nucl Med 21(4):209-215

55. Kwee TC, Takahara T, Ochiai R et al (2009) Whole-body diffusion-weighted magnetic resonance imaging. Eur J Radiol 70:409e17

56. Kwee TC, Takahara T, Ochiai R, Nievelstein RAJ, Luijten PR (2008) Diffusion weighted whole-body imaging with background body signal suppression (DWIBS): features and potential applications in oncology. Eur Radiol 18:1937-1952

57. Stone AJ, Browne JE, Lennon B, Meaney JF, Fagan AJ (2012) Effect of motion on the ADC quantification accuracy of wholebody DWIBS. MAGMA 25(4):263-266

58. Takahara T, Ogino T, Okuaki T et al (2007) Respiratory gated body diffusion weighted imaging avoiding prolongation of scan time: tracking only navigator echo (TRON) technique. Presented at the Joint Annual Meeting ISMRM-ESMRMB, Berlin, Germany, May 19-25, 2007

59. Herneth AM, Mayerhoefer M, Schernthaner R, Ba-Ssalamah A, Czerny C, Fruehwald-Pallamar J (2010) Diffusion weighted imaging: lymph nodes. Eur J Radiol 76(3):398-406

60. Adams HJ et al (2015) Opportunities and limitations of bone marrow biopsy and bone marrow FDG-PET in lymphoma. Blood Rev 29(6):417-425

61. Adams HJ et al (2016) Systematic review and meta-analysis on the prognostic value of complete remission status at FDG-PET in Hodgkin lymphoma after completion of first-line therapy. Ann Hematol 95(1):1-9

62. Lin C, Luciani A, Itti E et al (2010) Whole-body diffusionweighted magnetic resonance imaging with apparent diffusion coefficient mapping for staging patients with diffuse large B-cell lymphoma. Eur Radiol 20(8):2027-2038

63. Toledano-Massiah S, Luciani A, Itti E, Zerbib P, Vignaud A (2015) Whole-body diffusion-weighted imaging in hodgkin lymphoma and diffuse large b-cell lymphoma. Radiographics 35(3):747-764

64. Moses WW (2011) Fundamental limits of spatial resolution in PET. Nucl Instrum Methods Phys Res Sect A 648:S236-S240

65. Griffeth LK (2005) Use of PET/CT scanning in cancer patients: technical and practical considerations. In: Proceedings (Baylor University Medical Center) 18(4):321-330

66. King AD, Ahuja AT, Yeung DK et al (2007) Malignant cervical lymphadenopathy: diagnostic accuracy of diffusion-weighted MR imaging. Radiology 245(3):806-813

67. Koh DM, Blackledge M, Padhani AR, Takahara T, Kwee TC (2012) Whole-body diffusion-weighted MRI: tips, tricks, and pitfalls. AJR Am J Roentgenol 199(2):252-262

68. Koc Z, Erbay G (2014) Optimal b value in diffusion-weighted imaging for differentiation of abdominal lesions. J Magn Reson Imaging 40(3):559-566

69. Lin C, Itti E, Luciani A, Haioun C, Meignan M, Rahmouni A (2010) Whole-body diffusion-weighted imaging in lymphoma Cancer Imaging 10(1A):S172-S178 